\title{
Dynamics of charged particles in an adiabatic thermal beam equilibrium
}

\author{
Haofei Wei and Chiping Chen \\ Plasma Science and Fusion Center, Massachusetts Institute of Technology, Cambridge, Massachusetts 02139, USA
}

(Received 16 March 2010; published 4 February 2011)

\begin{abstract}
Charged-particle motion is studied in the self-electric and self-magnetic fields of a well-matched, intense charged-particle beam and an applied periodic solenoidal magnetic focusing field. The beam is assumed to be in a state of adiabatic thermal equilibrium. The phase space is analyzed and compared with that of the well-known Kapchinskij-Vladimirskij (KV)-type beam equilibrium. It is found that the widths of nonlinear resonances in the adiabatic thermal beam equilibrium are narrower than those in the KV-type beam equilibrium. Numerical evidence is presented, indicating almost complete elimination of chaotic particle motion in the adiabatic thermal beam equilibrium.
\end{abstract}

DOI: 10.1103/PhysRevSTAB.14.024201

PACS numbers: 29.27.-a, 05.45.Gg

\section{INTRODUCTION}

High-brightness beams in particle accelerators and beam devices and facilities are often generated in a regime where space charge plays an important role. Examples of highbrightness electron beams are rf and thermionic photoinjectors, thermionic DC injectors, electron beams in high-power rf sources, etc. Examples of high-brightness ion beams include negative hydrogen ion beams in spallation neutron sources and various ion beams in high energy density physics research.

Exploration of equilibrium states of charged-particle beams and their stability properties is critical to the advancement of basic particle accelerator physics. Of particular concern are emittance growth and beam losses, which are related to the evolution of charged-particle beams in their nonequilibrium states. To minimize emittance growth and control beam losses, it is critical to find and study equilibrium distributions of high-brightness charged-particle beams in accelerators and beam transport systems.

Several kinetic equilibria have been discovered for periodically focused intense charged-particle beams. Wellknown equilibria for periodically focused intense beams include the Kapchinskij-Vladimirskij (KV) equilibrium in an alternating-gradient (AG) quadrupole magnetic focusing field $[1,2]$ and the periodically focused rigid-rotor Vlasov equilibrium of the KV type in a periodic solenoidal magnetic focusing field $[3,4]$. Both of these beam equilibria [1-4] have a singular ( $\delta$-function) distribution in the four-dimensional phase space. Such a $\delta$-function distribution gives a uniform density profile across the beam in the transverse directions, and a transverse temperature profile which peaks on axis and decreases quadratically to zero on the edge of the beam. Because of the singularity in the distribution functions, these beam equilibria are not likely to occur in real physical systems and cannot provide realistic models for theoretical and experimental studies and simulations except in the zero-temperature limit. For example, the KV equilibrium model cannot be used to explain the beam tails in the radial distributions observed in recent high-intensity beam experiments [5]. Recently, adiabatic thermal beam equilibria have been discovered in a periodic solenoidal magnetic focusing field [6-8] and an AG quadrupole magnetic focusing field $[8,9]$. The measured density distribution [5] matches that of the adiabatic thermal beam equilibrium in a spatially varying solenoidal magnetic focusing field $[6,8]$.

There have been many studies of charged-particle dynamics in the KV-type equilibria [10-14]. These studies have shown that the phase space for the KV-type equilibria exhibits rich nonlinear resonances and chaotic seas for charged particles outside the beam envelope [10,11]. If charged particles cross the beam envelope due to perturbations, they may enter chaotic seas to form a beam halo or cause beam losses [12-14].

The purpose of this paper is to show numerically that chaotic particle motion is almost completely eliminated in the adiabatic thermal beam equilibrium. The importance of this result is twofold: First, the elimination of chaotic particle motion provides a further numerical proof that the scaled transverse Hamiltonian defined in Eq. (25) in [6] is a very good approximate constant of motion. This approximate constant of motion and the exact contact of motion of the canonical angular momentum assure that the motion of charged particles is approximately integrable in the fourdimensional phase space of the adiabatic thermal beam equilibrium. Second, the elimination of chaotic particle motion may provide valuable insight into how to control chaotic particle motion, halo formation, and beam loss in beam transport channels. In particular, if adiabatic thermal beams are stable, they may be free from chaotic particle motion, beam halo formation, and beam loss.

The organization of the present paper is as follows. In Sec. II, the basic equations of the adiabatic thermal beam equilibrium are reviewed. The equations of motion for a charged particle are presented. In Sec. III, results of a numerical analysis of charged-particle motion are 
discussed. In particular, the Poincare surface-of-section maps of the motion of charged particles in phase space are generated numerically. Comparisons are made between the KV-type and adiabatic thermal beam equilibria. Numerical evidence is presented for almost complete elimination of chaotic particle motion in the adiabatic thermal beam equilibrium. In Sec. IV, the conclusion of the present paper is presented.

\section{THEORETICAL MODEL}

We study charged-particle dynamics in the adiabatic thermal equilibrium of an intense charged-particle beam propagating with constant axial velocity $\beta_{b} c \hat{\mathbf{e}}_{z}$ in the periodic solenoidal magnetic focusing field,

$$
\mathbf{B}^{\mathrm{ext}}=B_{z}(s) \hat{\mathbf{e}}_{z}-\frac{1}{2} \frac{d B_{z}(s)}{d s}\left(x \hat{\mathbf{e}}_{x}+y \hat{\mathbf{e}}_{y}\right),
$$

where $s=z$ is the axial coordinate, $B_{z}(s+S)=B_{z}(s)$ is the axial magnetic field, $S$ is the fundamental periodicity length of the focusing field, and $c$ is the speed of light in vacuum. The adiabatic thermal beam equilibrium has been derived under the paraxial approximation with the following assumptions: (1) $r_{b \mathrm{rms}} \ll S$, where $r_{b \mathrm{rms}}$ is the rms beam radius and (2) $\nu / \gamma_{b}^{3} \beta_{b}^{2} \ll 1$, where $\nu=q^{2} N_{b} / m c^{2}$ is the Budker parameter of the beam, $q$ and $m$ are the particle charge and rest mass, respectively, $N_{b}=$ $\int_{0}^{\infty} n_{b}(r, s) 2 \pi r d r=$ const is the number of particles per unit axial length, and $\gamma_{b}=\left(1-\beta_{b}^{2}\right)^{-1 / 2}$ is the relativistic mass factor.

In the adiabatic thermal beam equilibrium [6-8], the beam density distribution is given by

$$
\begin{aligned}
n_{b}(r, s)= & \frac{4 \pi C \varepsilon_{\mathrm{th}}^{2}}{r_{b \mathrm{rms}}^{2}(s)} \exp \left\{-\left[\frac{K}{2}+\frac{4 \varepsilon_{\mathrm{th}}^{2}}{r_{b \mathrm{rms}}^{2}(s)}\right] \frac{r^{2}}{4 \varepsilon_{\mathrm{th}}^{2}}\right. \\
& \left.-\frac{q}{\gamma_{b}^{2} k_{B} T_{\perp}(s)} \phi(r, s)\right\},
\end{aligned}
$$

and the self-electric potential $\phi(r, s)$ is determined by the Poisson equation,

$$
\frac{1}{r} \frac{\partial}{\partial r}\left(r \frac{\partial \phi}{\partial r}\right)=-4 \pi q n_{b}(r, s)
$$

and the free-space boundary conditions. In Eqs. (2) and (3), $C$ is a constant determined by $N_{b}=\int_{0}^{\infty} n_{b}(r, s) 2 \pi r d r$, $K \equiv 2 q^{2} N_{b} / \gamma_{b}^{3} m \beta_{b}^{2} c^{2}$ is the generalized beam perveance, $\varepsilon_{\mathrm{th}}=\left[k_{B} T_{\perp}(s) r_{b \text { rms }}^{2} / 2 \gamma_{b} m \beta_{b}^{2} c^{2}\right]^{1 / 2}$ is the rms thermal emittance in the Larmor frame, $\tilde{x}=x \cos \varphi-y \sin \varphi$ and $\tilde{y}=x \sin \varphi+y \cos \varphi$, where $\varphi=\int_{0}^{s} \sqrt{\kappa_{z}(s)} d s, T_{\perp}(s)$ is the Kelvin temperature of the beam, $k_{\mathrm{B}}$ is the Boltzmann constant, and the rms beam envelope $r_{b \text { rms }}(s)=r_{b \text { rms }}(s+S)$ solves the beam envelope equation

$$
\frac{d^{2} r_{b \mathrm{rms}}}{d s^{2}}+\kappa_{z}(s) r_{b \mathrm{rms}}-\frac{K}{2 r_{b \mathrm{rms}}}=\frac{4 \varepsilon_{\mathrm{th}}^{2}}{\left(1-\omega_{b}^{2}\right) r_{b \mathrm{rms}}^{3}},
$$

where $\sqrt{\kappa_{z}(s)} \equiv q B_{z}(s) / 2 \gamma_{b} m \beta_{b} c^{2}$ and $\omega_{b}^{2}=1-\left(\varepsilon_{\text {th }} /\right.$ $\left.\varepsilon_{\tilde{x} \mathrm{rms}}\right)^{2}$ with $\varepsilon_{\tilde{x} \mathrm{rms}}$ being the rms emittance in the $\tilde{x}$ direction.

\section{NUMERICAL ANALYSIS}

Figure 1 shows (a) normalized focusing parameter $S^{2} \kappa_{z}(s)$ and (b) normalized rms envelope $r_{b \text { rms }} / \sqrt{4 \varepsilon_{\mathrm{th}} S}$ versus the normalized distance $s / S$ for the choice of system parameters corresponding to $S \sqrt{\kappa_{z}(s)}=$ $(2 / 3)^{1 / 2} \sigma_{0}[1+\cos (2 \pi s / S)], \quad S K / 4 \varepsilon_{\text {th }}=7.0, \quad \omega_{b}=0$, and vacuum phase advance $\sigma_{0}=80^{\circ}$. For $\omega_{b}=0, \varepsilon_{\mathrm{th}}=$ $\varepsilon_{\tilde{x} \mathrm{rms}}$ and the KV-type and adiabatic thermal beam equilibria have the same rms beam envelopes.

Figure 2 shows (a) density $n_{b}$ and (b) radial self-electric field $E_{r}$ for the KV-type and adiabatic thermal beam equilibria at $s=0$ with the same choice of system parameters as in Fig. 1. While the self-electric fields of the two beams are similar, there are three important differences: (a) the density in the interior for the adiabatic thermal beam is
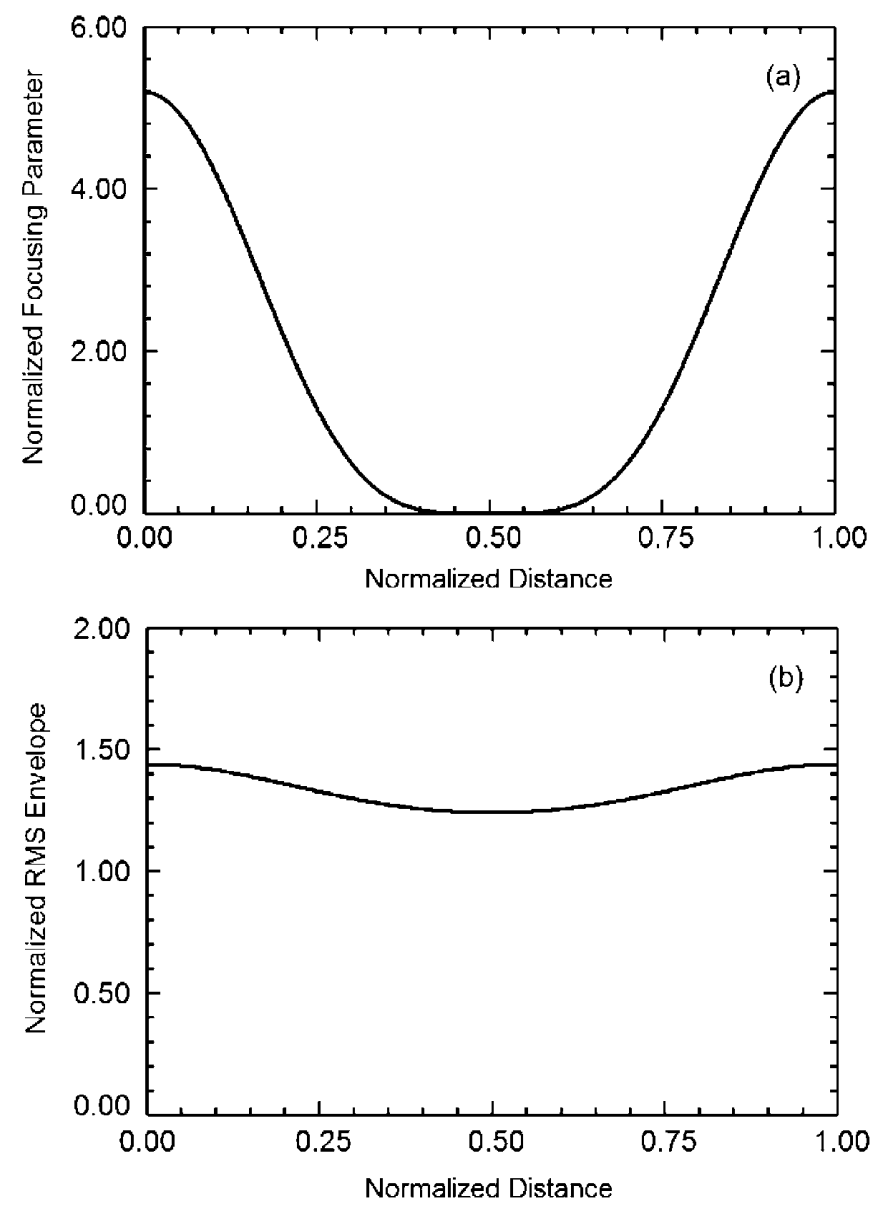

FIG. 1. Plots of (a) normalized focusing parameter $S^{2} \kappa_{z}(s)$ and (b) normalized rms envelope $r_{b \text { rms }} / \sqrt{4 \varepsilon_{\mathrm{th}} S}$ versus the normalized distance $s / S$ for the choice of system parameters corresponding to $S K / 4 \varepsilon_{\text {th }}=7.0, S \sqrt{\kappa_{z}(s)}=(2 / 3)^{1 / 2} \sigma_{0}[1+$ $\cos (2 \pi s / S)], \omega_{b}=0$, and $\sigma_{0}=80^{\circ}$. 

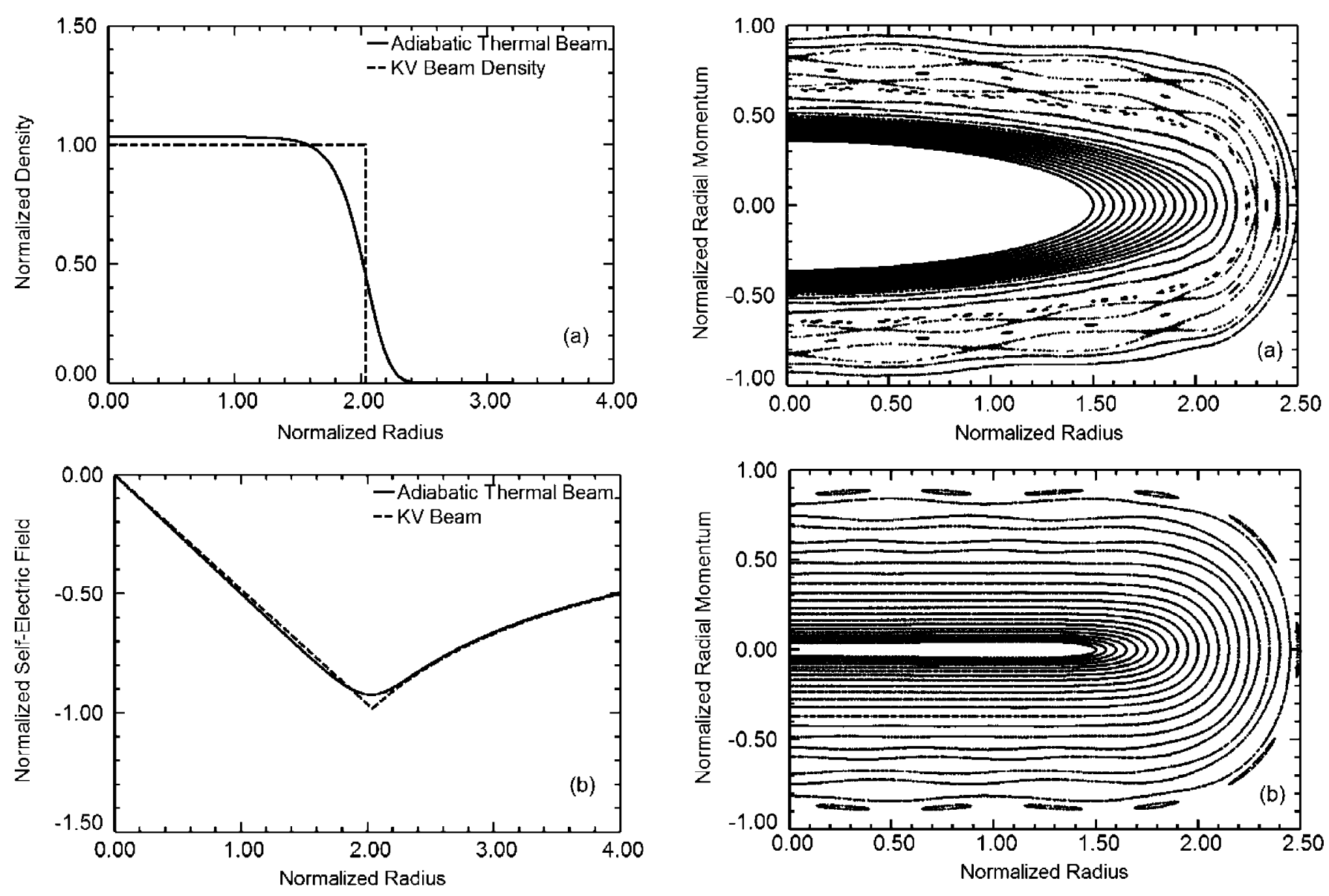

FIG. 2. Plots of (a) normalized density $n_{b} / n_{\mathrm{KV}}(0,0)$ and (b) normalized radial self-electric field $S^{3 / 2} K E_{r} / 4 \varepsilon_{\text {th }}^{1 / 2} q N_{b}$ versus normalized radius $r / \sqrt{4 \varepsilon_{\mathrm{th}} S}$ in the KV-type beam equilibrium (dashed curve) and the adiabatic thermal beam equilibrium (solid curve) at $s=0$ for the same choice of system parameters as in Fig. 1. Here, $n_{\mathrm{KV}}(0,0)$ is the density of the $\mathrm{KV}$-type beam equilibrium at $s=0$ and $r=0$.

higher than that for the KV-like beam; (b) the electric field near the normalized radius $r / \sqrt{4 \varepsilon_{\mathrm{th}} S} \approx 2.0$ has a smooth transition from negative to positive slope for the adiabatic thermal beam, whereas its radial derivative is discontinuous for the KV-type beam equilibrium; (c) the self-electric field near the edge of the adiabatic thermal beam is weaker than that of the KV-type beam. These differences result in significant changes in charged-particle dynamics (see Figs. 3 and 4).

The radial equation of motion of a charged particle in the cylindrical coordinates is

$$
\frac{d^{2} r}{d s^{2}}+\frac{P_{\theta}^{2}}{r^{3}}+\kappa_{z}(s) r+\frac{q}{\gamma_{b}^{3} m \beta_{b}^{2} c^{2}} \frac{\partial \phi(r, s)}{\partial r}=0
$$

where the canonical angular momentum $P_{\theta}$ is conserved. Figure 3 shows a comparison between the Poincaré surface-of-section maps of charged-particle trajectories in (a) KV-type beam equilibrium and (b) adiabatic thermal

FIG. 3. Poincaré surface-of-section maps of charged-particle trajectories in (a) KV-type beam equilibrium and (b) adiabatic thermal beam equilibrium for $P_{\theta}=0$ and the same choice of system parameters as in Fig. 1. Here, the normalized radial momentum is $\left(S / 4 \varepsilon_{\text {th }}\right)^{1 / 2} d r / d s$ and the normalized radius is $r / \sqrt{4 \varepsilon_{\mathrm{th}} S}$.

beam equilibrium for the choice of system parameters corresponding to $P_{\theta}=0, \quad S \sqrt{\kappa_{z}(s)}=(2 / 3)^{1 / 2} \sigma_{0}[1+$ $\cos (2 \pi s / S)], \quad \sigma_{0}=80^{\circ}, \quad \omega_{b}=0$, and $S K / 4 \varepsilon_{\text {th }}=7.0$. These parameters correspond to a highly space-chargedominated beam rotating at the (local) Larmor frequency in a high-strength periodic solenoidal magnetic focusing field. They are generated by plotting $\left(r, P_{r}\right)$ as a trajectory arrives at the lattice points $s / S=0,1,2, \ldots, 2000$. For these parameters, the density for the KV-type beam equilibrium drops abruptly at $r / \sqrt{4 \varepsilon_{\mathrm{th}} S} \approx 2.0$, whereas the density for the adiabatic thermal beam equilibrium falls from its flattop value to almost zero between $r / \sqrt{4 \varepsilon_{\mathrm{th}} S} \cong$ 1.6 and 2.4 .

For $r / \sqrt{4 \varepsilon_{\text {th }} S}<2.0$, the phase space is regular in both the KV-type and adiabatic thermal beam equilibria, and the action of a charged particle in the KV-type beam is larger than that in the adiabatic thermal beam, as shown in Fig. 3. The phase space area (action) of a charged particle in the interior of the adiabatic thermal beam is significantly smaller than that of the KV-type beam because the density 

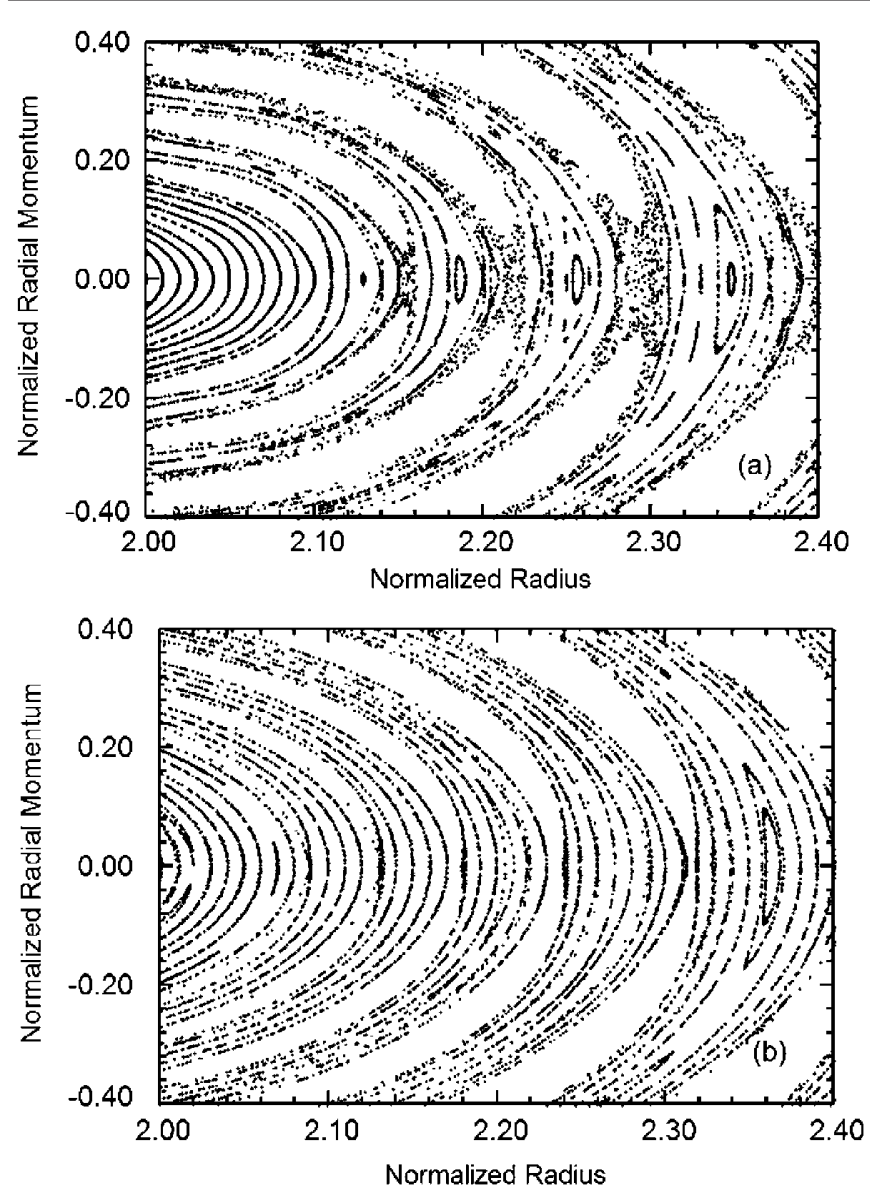

FIG. 4. Close-ups of Poincaré surface-of-section maps of charged-particle trajectories for the two cases shown in Fig. 3.

of the adiabatic thermal beam approaches the density of the corresponding cold beam, which is higher than the density of the KV-type beam. In the region $2 \leq r / \sqrt{4 \varepsilon_{\mathrm{th}} S} \leq 2.4$, however, there are striking differences between the $\mathrm{KV}$-type and adiabatic thermal beam equilibria, as shown in Fig. 4. Comparing Fig. 4(a) with Fig. 4(b), there are two important differences to note. First, there are chaotic seas in the phase space of the KV-type beam, whereas chaotic motion is almost absent in the phase space of the adiabatic thermal beam equilibrium. Second, the widths of the nonlinear resonances in the adiabatic thermal beam equilibrium are narrower than those in the $\mathrm{KV}$-type beam equilibrium. For example, the width of the nonlinear resonance at $\left(r / \sqrt{4 \varepsilon_{\mathrm{th}} S}, P_{\theta}\right) \cong(2.36,0)$ is $\Delta r / \sqrt{4 \varepsilon_{\mathrm{th}} S}=$ 0.013 in the adiabatic thermal beam, whereas the width of the corresponding resonance in the KV-type beam is $\Delta r / \sqrt{4 \varepsilon_{\mathrm{th}} S}=0.021$.

\section{CONCLUSION}

We analyzed charged-particle motion in the self-electric and self-magnetic fields of a well-matched, intense charged-particle beam in a period solenoidal magnetic focusing field. We assumed that the beam is in the state of adiabatic thermal equilibrium. We compared the phase space of the adiabatic thermal beam equilibrium with that of a corresponding KV-type beam equilibrium. We found that the widths of some of the nonlinear resonances in the adiabatic thermal beam equilibrium are narrower than those in the KV-type beam equilibrium. We presented numerical evidence for almost complete elimination of chaotic particle motion in the adiabatic thermal beam equilibrium in a periodic solenoidal magnetic focusing field.

\section{ACKNOWLEDGMENTS}

The authors wish to thank Dr. Ksenia R. Samokhvalova and Dr. Jing Zhou for helpful discussions. This work was supported by U.S. Department of Energy, Office of High Energy Physics, Grant No. DE-FG02-95ER40919, and Office of Fusion Energy Science, Grant No. DE-FG0205ER54835. Research by H. Wei was also supported by Massachusetts Institute Technology's Undergraduate Research Opportunity Program.

[1] I. M. Kapchinskij and V. V. Vladimirskij, in Proceedings of the Conference on High Energy Accelerators (CERN, Geneva, 1959), p. 274.

[2] F. J. Sacherer, Transverse Space-Charge Effects in Circular Accelerators (Lawrence Berkeley National Laboratory, Berkeley, California, 1968).

[3] C. Chen, R. Pakter, and R. C. Davidson, Phys. Rev. Lett. 79, 225 (1997).

[4] R.C. Davidson and H. Qin, Physics of Intense Charged Particle Beams in High Energy Accelerators (Imperial College Press, Singapore, 2001), p. 242.

[5] S. Bernal, B. Quinn, M. Reiser, and P. G. O'Shea, Phys. Rev. ST Accel. Beams 5, 064202 (2002).

[6] J. Zhou, K. R. Samokhvalova, and C. Chen, Phys. Plasmas 15, 023102 (2008).

[7] K. R. Samokhvalova, J. Zhou, and C. Chen, Phys. Plasmas 14, 103102 (2007).

[8] K. R. Samokhvalova, Ph.D. Thesis, Massachusetts Institute of Technology, 2008.

[9] K. R. Samokhvalova, J. Zhou, and C. Chen, Phys. Plasmas 16, 043115 (2009).

[10] J.-M. Lagniel, Nucl. Instrum. Methods Phys. Res., Sect. A 345, 46 (1994).

[11] C. Chen, R. Pakter, and R. C. Davidson, Phys. Plasmas 6, 3647 (1999).

[12] Q. Qian, R.C. Davidson, and C. Chen, Phys. Rev. E 51, R5216 (1995).

[13] Y. Fink, C. Chen, and W. P. Marable, Phys. Rev. E 55, 7557 (1997).

[14] J. Zhou, B.-L. Qian, and C. Chen, Phys. Plasmas 10, 4203 (2003). 